\title{
Application of Red Pulsed Laser Therapeutic Radiation for Healing Larynx Bilateral Paralysis After Stringectomy
}

\author{
Zhanna Evgenevna Komarova, Alexey Nicolaevich Nasedkin, Vitalyi Nicolaevich Selin, \\ Valentin Yurievich Tyukin, Anna Yurievna Polschikova \\ Department of Otorhinolaryngology, Moscow Regional Scientific Research Clinical Institute of M. F. Vladimirsky, Moscow, Russia
}

Email address:

zhkomarova@icloud.com (Z. E. Komarova)

To cite this article:

Zhanna Evgenevna Komarova, Alexey Nicolaevich Nasedkin, Vitalyi Nicolaevich Selin, Valentin Yurievich Tyukin, Anna Yurievna Polschikova. Application of Red Pulsed Laser Therapeutic Radiation for Healing Larynx Bilateral Paralysis After Stringectomy. International Journal of Otorhinolaryngology. Vol. 4, No. 1, 2018, pp. 27-30. doi: 10.11648/j.ijo.20180401.17

Received: July 10, 2018; Accepted: July 30, 2018; Published: August 27, 2018

\begin{abstract}
The study aim was to increase the effectiveness of surgical treatment and rehabilitation of patients with bilateral paralysis of the larynx by applying low-intensity laser radiation of the visible red spectrum in the pulsed mode in this category of patients in the postoperative period. There were healed 18 female patients with bilateral paralysis of the larynx, 14 of them was tracheotomized, 4 of them - wasn't. According to the method of surgical intervention, the patients were divided into 2 groups. The first one includes 9 female patients who had chordaritenoidotomy with coblator. The second one includes 9 female patients who had chordaritenoidotomy with microsyrgery tools. All of patients had antiphlogistic therapy after operation, 5 patients from each group also had additionally low-intensity laser radiation therapy with device «Mustang 2000». On the third and tenth day patients had Doppler laser flowmetry, considering variation coefficient $(\mathrm{Kv})$. On the third day 4 patients from each group (who haven't additionally red pulsed laser therapy) have $\mathrm{Kv}=9,10$ patients (who have additionally red pulsed laser therapy) $-\mathrm{Kv}=13$. On the 10 day the first ones had $\mathrm{Kv}=26$, the second ones $-\mathrm{Kv}=32$. The criteria for the effectiveness of postoperative treatment were the quality and timing of wound healing, the absence of cicatricial measurements in the long-term postoperative period, and the normalization of respiratory function. After 4 months both groups have such complications as cicatricial stenosis of vocal folds and underfolds area, polypus of vocal folds. In 10 patients receiving laser therapy, no complications were observed. In conclusion it can be said that the method of surgical intervention (in the first group chordaritenoidotomy with the cold-plasma apparatus cobblator, in the second group - chordaritenoidotomy with instruments for microsurgery of the larynx) practically does not affect the final result. Laser therapy is an important factor in the activation of regenerative processes in the postoperative area.
\end{abstract}

Keywords: Bilateral Paralysis of the Larynx, Chordarinoidotomy, Low-Intensity Laser Radiation

\section{Introduction}

Chronic laryngeal stenosis is a syndrome, the end result of the presence in the larynx of many pathological processes that differ in origin, pathogenesis and clinical manifestations, which constitute a complex, mosaic pattern [1]. With increasing specific gravity of iatrogenic lesions, the problem of chronic laryngeal stenoses appeared in a new perspective. Volumetric operations on the neck and mediastinal organs often cause a violation of innervation of the larynx, leading to paralytic stenosis. This often happens not because of technical errors, but because of the prevalence of the pathological process. The recurrent nerve during surgery on the thyroid gland is stretched and wounded in about $1.5-5 \%$ of cases [2]. And with atypical and complicated repeated strumectomy, it is already injured in $10-12 \%$ of cases.

Surgical treatment of paralytic stenosis of the larynx can be undertaken in the short term after its occurrence in the event that it was evident that the two return nerves were severed during extirpation of the thyroid gland for a malignant tumor, resection of the trachea, esophagus, neck injury, etc. If there was no obvious violation of the integrity 
of the recurrent nerves, reconstructive operations on the larynx are carried out not earlier than in 6 months, it is during this period of time that the restoration of the mobility of the vocal fold can proceed in the case of temporary disruption of the nerve function in case of trauma [1]. During this period, tracheostomy is applied to ensure adequate breathing or endoscopic laterofixation of the vocal fold. In the postoperative period, conservative therapy is performed to improve the recovery of nerve function.

About one hundred years ago, the first methods of treating paralytic stenoses of the larynx were proposed by Killian, and then by A. F. Ivanov. Since then, there has been a continuous search for reliable and less traumatic surgical methods of treatment with the use of more advanced materials for prosthetics and new surgical instruments.

The difficulty in choosing the optimal method for the surgical treatment of paralytic stenoses is due to the fact that with the immobility of the vocal folds, a contradiction arises: the better the voice, the worse the respiratory function is provided. But the operation, which significantly widens the respiratory cleft due to its posterior parts, can cause disruption of the separation function, which means that saliva enters the respiratory tract.

Restoration of respiratory function in patients with paralytic stenosis of the larynx can be achieved after external laryngotracheal plastic surgery, as well as endoscopic methods. In recent years, endoscopic chordectomy, aritenoidectomies, chordaritenoidotomy performed by microinstruments or surgical laser have been successfully used. D. G. Chireshkin (1994), the insufficient effect of a chordectomy is explained by the sucking action of the air jet (Bernoulli phenomenon), and in this connection a roller is formed on the site of the removed voice fold, again narrowing the voice gap. The method of unilateral submucosal chordaritenoidectomy was proposed in 1968 by Kleinsasser. Later it was modified in 1980 by Y. Preobrazhensky, and later in 1995 by A. E. Uskov. The most effective surgical intervention in the treatment of bilateral paralysis of the larynx is chordaritenoidotomy, which allows to maintain a satisfactory voice function and guarantees the absence of aspiration syndrome. With chordaritenoidotomy, the body does not affect the body, the lateral cricoid muscle, the space Pressman, which is of great importance for the favorable course of the postoperative period and the reduction of the risk of gross scarring in the area of surgical intervention.

The final result of surgical treatment of laryngeal paralysis is significantly influenced by correct preoperative preparation and postoperative drug therapy. Surgical interventions can be performed only after correcting the level of thyroid hormones. Thus it is necessary to pay attention to the presence of pharyngo-laryngeal reflux. The paramedic position of even one of the vocal folds is a prerequisite to throwing acidic contents into the larynx, which inevitably causes a spastic reaction of the larynx. In this regard, it is necessary to prevent pharyngo-laryngeal reflux by diet and special diet (a split meal, a ban on taking a horizontal position for 4-5 hours after eating) [1].

Restoration of nerve conduction is also an important point in the treatment of peripheral paresis of the larynx. For this purpose, usually use anticholinesterase drugs (proserine) and M-cholinolytics (metacin).

In modern surgery, the search for new methods of local influence on the wound process does not stop, with the help of which it is possible to shorten the periods of rehabilitation of patients. Features of the inflammatory-reparative process in the larynx and laryngeal tracheal department are determined by the specific features of the anatomical structure of the hollow organs of the neck, as well as the complex structural interactions of various tissues of the anterior part of the neck: skin, muscles, cartilage, fascia, mucosa [3]. These tissues differ not only in their diverse morphological organization, but also in their different responses to damage and their individual ability for reparative processes. Thus, when the wound is healed after surgery on the larynx, it is very important that adequate healing is combined with the absence of excessive scarring, which inevitably leads to restenosis and negates the results of surgical intervention [4].

Laser therapeutic procedures conducted by a course of 810 sessions, both before the operation for the prevention of infiltration and suppuration, and after surgery, improve local capillary blood circulation, metabolic processes, oxygenation and trophic tissue, which stabilizes the entire postoperative period and at times reduces the likelihood of postoperative complications. LILR (low-intensity laser radiation) stimulates the activity of the most important bioenergetic enzymes (dehydrogenase, cytochrome oxidase), catalase, aldolase, ATPase, acid and alkaline phosphatase, which contributes to the preservation or restoration of homeostasis and adaptation of the organism to stress conditions [5, 6, 7].

Under the influence of LILR, a change in the physiology of tissues is possible both in the direction of strengthening and in the direction of inhibition of their metabolism, depending on the initial state of the organism and the dose of exposure, which leads to the attenuation of pathological processes, the normalization of physiological reactions, and the restoration of the regulatory functions of the nervous system [3, 8]. Laser therapy with proper application allows the body to restore the disturbed systemic balance. It is known that LILR is able to activate the functional state of the cellular and humoral units of immunity, which is manifested by the elimination of various kinds of defects and disorders in the immunity system, the normalization of defense mechanisms at the local and systemic levels.

As for laser therapy, it has proven itself in the best way and in the impact on the wound process [9]. Therefore, in this paper there was used the laser radiation of the visible red spectrum (wavelength $0.63 \mu \mathrm{m}$ ), and in the pulsed mode, since in the literature there appeared information that exactly such laser radiation (red pulsed) is the most effective in the therapy of inflammation in general and acceleration of the processes of tissue regeneration in the wound process, in particular $[10,11,12]$. 


\section{Objective}

Increase the effectiveness of surgical treatment and rehabilitation of patients with bilateral paralysis of the larynx by applying low-intensity laser radiation of the visible red spectrum in the pulsed mode in this category of patients in the postoperative period.

\section{Materials and Methods}

In the ENT department of MONIKI under medical supervision 18 women with bilateral paralysis of the larynx (vocal folds in the position of reduction) were treated, after a strukectomy for multinodular goiter. Duration of the disease was from 10 months to 18 years, age from 35 to 65 years. To clarify the diagnosis, fibrolaringoscopy was used. At the place of residence, 7 patients were tracheotomized. In the ENT department for stenosis of the larynx of the 2nd degree, 7 more patients were tracheotomized. In 4 patients, tracheotomy was not performed; respiration at rest in them was compensated, which was confirmed by data of fibrolaringoscopy and stroboscopy, as well as by the results of the study of the function of external respiration.

According to the method of surgical intervention, the patients were divided into 2 groups. The first group consisted of 9 patients, who had a coblator with the cold-plasma apparatus, using chondaritenoidotomy. In the second group 9 patients with chordaritenoidotomy were performed with instruments for microsurgery of the larynx. All patients in the first stage underwent general anesthesia with direct microlaringoscopy and chordaritenoidotomy. Patients of the second group had a mucosal incision at the border of the middle and posterior third of the vocal fold, the line of the incision was directed outwards and downwards, arching around the vocal appendage of the arytenoid cartilage. The resected fragment was fixed with forceps and cut off. Operative interventions in most cases were bloodless. This method of operation provided a satisfactory voice function at the expense of the preserved anterior sections of the vocal folds and a significant improvement in the respiratory function. Long-term results of the operation in most cases were better than in the immediate time after it. With the passage of time, the laterofixation of the remaining part of the voice fold with the scar occurred and some pulling it upward, which increased the clearance of the glottis. The final picture of the lumen of the glottis was formed 3-4 months later, and sometimes 6 months after the operation. In both groups there were 2 untreated patients.

Patients in the groups were comparable in age, location, prevalence of the pathological process and the presence of concomitant diseases.

All 18 patients underwent essentially the same type of antibacterial, anti-edematous and anti-inflammatory therapy, but 5 patients in each group, randomized before the operation, were additionally treated with laser therapy along with the above treatment. There were used the Mustang 2000 apparatus using a laser head generating in the red spectrum with a wavelength of 0.65-0.67 $\mu \mathrm{m}$, in a pulse-periodic mode, with a frequency of $80 \mathrm{~Hz}$. The average time of laser exposure is 5 minutes. At the same time, the energy density (ED) on average was $1.5-3.0 \mathrm{~J} / \mathrm{cm} 2$. The number of sessions is $8-10$ per course of treatment. The optimal depth of penetration of such laser radiation is $0.5-3 \mathrm{~cm}$, depending on the nature of the biological tissue. Laser exposure was exposed to the skin of the anterior surface of the neck in the projection region of the cartilages of the larynx (projection scanning mode of action). The output power from the laser head was monitored before each session, and the laser therapy session was tried at the same time ( \pm 1 hour).

To objectify the evaluation of the effectiveness of laser therapy, laser doppler flowmetry (LDF) was performed on all patients on the 3rd and 10th days after the operation using the LAKK 01 laser analyzer with a single-channel skin sensing option in the projection of the larynx using a skin sensor. When evaluating the results of LDF, it was necessery to be guided by the coefficient of variation $(\mathrm{Kv})$. A higher value of this indicator indicated an improvement in microcirculation and the appearance of new capillaries in the region of the skin exposed to the laser.

The criteria for the effectiveness of postoperative treatment were the quality and timing of wound healing, the absence of cicatricial measurements in the long-term postoperative period, and the normalization of respiratory function.

\section{Results}

Comparison of the results of treatment in the control groups of patients showed that on the 3rd day after chordaritenoidotomy in 8 patients (4 out of groups I and II) who did not undergo laser therapy, in the postoperative period, according to LDF data, $\mathrm{Kv}$ averaged 9 units, Fibrolaringoscopy revealed pronounced edema and hyperemia of the postoperative area. While in 10 patients who received laser therapy, Kv averaged 13 units. This indicated an increase in the degree of neovascularization, i.e. the appearance of new capillaries after laser exposure. When fibrolaringoscopy, a small swelling and flushing in the postoperative area was noted, while breathing with a closed tracheotomy tube was compensated.

On the 10th day of $\mathrm{Kv}$ in 8 patients not receiving laser therapy, an average of 26 units, while fibro-laryngosks in the area of the postoperative wound, there was hyperemia and a pronounced thickening of one third of the voice fold. These phenomena stopped at 13-14 days after surgical treatment. In 10 patients receiving laser therapy in the postoperative period, Kv was 32 units, i.e. 6 units higher. When fibrolaringoscopy, they did not observe the phenomena of inflammation in the postoperative area, subjectively patients noted improved respiration through the natural airways.

And in the first and second groups in the remote postoperative period (after 3-4 months), the following complications were observed:

in the first group (operated by the cobblator), one patient showed cicatricial obstruction of the lining of the larynx, 
which led to laryngotracheal plastic surgery and the installation of a T-tube; two patients developed cicatrical changes at the level of the vocal folds, which caused repeated surgical intervention.

In the second group (instruments for microsurgery), one patient had a polyp in the anterior third of the left vocal fold, which was subsequently removed, and the other patient had cicatrical changes in the vocal folds, which also resulted in repeated intervention on the larynx.

In all patients of both groups, who underwent laser therapy in the postoperative period, no complications were observed.

\section{Conclusion}

The method of surgical intervention (in the first group chordaritenoidotomy with the cold-plasma apparatus cobblator, in the second group - chordaritenoidotomy with instruments for microsurgery of the larynx) practically does not affect the final result.

The application of the red pulsed therapeutic laser accelerated the healing process of the postoperative wound by an average of 3 days, due to the increased microcirculation, which is confirmed by laser Doppler flowmetry data, and this in turn made it possible to avoid restenosis in these patients in the long postoperative period.

\section{Findings}

Laser therapy in patients who underwent chordaritenoidotomy for bilateral paralysis of the larynx is an important factor in activating regenerative processes in the postoperative area due to an increase in the number of new capillaries in the tissues of the operating zone (based on laser Doppler flowmetry data), which thereby shortens the healing time wound for an average of 3 days, increases the effectiveness of traditional conservative treatment and prevents the occurrence of restenosis.

\section{References}

[1] Pluzhnikov MS, Ryabova MA, Karpishchenko S. A. Chronic stenoses of the larynx St. Petersburg "Esculap", 2004. - 206s.

[2] Vasilenko, Yu. S. Iatrogenic dysphonia and their treatment / Yu. S. Vasilenko, G. Kittel // XVI congress of otorhinolaryngologists of the Russian Federation: theses. doc. Sochi, 2001.- C. 454-458.

[3] Zenger VG, Nasedkin AN, Parshin VD Surgery of larynx and trachea injuries. - M.: Medkniga Publishing House, 2007. 348 p.
[4] Lapchenko AS, Gurov AV, Malchenko OV Kucherov AG, Lapchenko AA Treatment of purulent-septic complications of inflammatory diseases of ENT organs using photodynamic therapy. Modern achievements of laser medicine and their application in practical public health". Materials of the scientific-practical conference with international participation dedicated to the 20th anniversary of the State Scientific Center of Laser Medicine of the Ministry of Health of the Russian Federation on 5-6 October 2006, Moscow. 150 p.

[5] Krechina EK, Maslova VV, Shidova A. V. and others. A comparative assessment of the effect of low-intensity pulsed and continuous laser radiation of red and infrared spectral ranges on microcirculation in the complex therapy of chronic periodontitis. Laser medicine. - 2009. - Т.13, вып.2. - P.22-26.

[6] Leiderman NE, Kochetkov AV, Moskvin S. V. LAZMIK technology in complex regenerative treatment of patients with chronic cerebral ischemia. Bulletin of new medical technologies. - 2009. - №4. - P. 104-106.

[7] Moskvin SV, Achilov AA Basics of laser therapy. - M.-Tver: Triad, 2008. $-256 \mathrm{p}$.

[8] Zenger V. G., Nasedkin A. N. Modern technologies in the treatment of diseases of the ear, throat and nose. - M.: Medkniga Publishing House, 2008. - 335c.

[9] Nasedkin A. N., Zenger V. G. Optimization of laser therapy methods for ear diseases. Throat and nose. Laser medicine. 2000. - T.4, №.4. -.9-12 p.

[10] Moskvin SV, Nasedkin AN, Kochetkov AV and others. Therapy with matrix pulsed lasers of the red radiation spectrum. - Tver: The Triad, 2007. - $112 \mathrm{p}$.

[11] Moskvin SV, Nasedkin AN, Osin A. Ya., Khan M. Ya. Laser therapy in pediatrics. - Moscow: Izd. EKSMO, 2010. - 479p.

[12] Moskvin SV, Efficiency of laser therapy. Series "Effective laser therapy". T. 2.- M.- Tver: OOO "Publishing House" Triada ", 2014.-896 p.

[13] Karpova O. Yu. Clinic, diagnosis and treatment of vocal and respiratory disorders in functional and organic diseases of the larynx: Author's abstract. diss. Dr. med. sciences. - M., 2001. $45 \mathrm{p}$.

[14] Kozlov VI, Builin V. A. Laser therapy with application of ALT "Mustang". - M.: Firma "Technika", 1998. - 148s.

[15] Moskvin S. V. Pulsed electromagnetic fields in physiotherapy. Problems of biophysical medicine. - Saratov: The Sarat Publishing House. honey. University, 2003. - P. 66-73.

[16] Uskov AE Endoscopic laryngoplasty in rehabilitation of patients with bilateral paralytic stenoses of the larynx: the author's abstract of the dissertation.... Candidate of Medical Sciences: 14.00.04 / St. Petersburg. NII of the ear, throat, nose and speech. - St. Petersburg, 1995. - 22 p.

[17] Yunina AI Trauma of the neck organs and their complications [Text]. - Moscow: Medicine, 1972. - 208 p. 